\title{
NOUVELLe
}

\section{Tension corticale de l'ovocyte et euploïdie : la juste mesure}

Isma Bennabi, Marie-Hélène Verlhac, Marie-Emilie Terret
$>$ Chez les eucaryotes, il existe deux types de divisions cellulaires: la mitose, qui concerne les cellules somatiques ( $2 n$ chromosomes), et la méiose, qui produit les gamètes haploïdes (n chromosomes), ovocytes chez la femelle et spermatozoïdes chez le mâle. La reproduction sexuée repose sur la fusion de ces deux gamètes haploïdes, conduisant à la formation d'un embryon. Étonnamment pour une stratégie reproductive, la méiose chez la femme est fortement sujette aux erreurs de ségrégation des chromosomes. Au moins $10 \%$ des grossesses humaines produisent des embryons aneuploïdes suite à des défauts méiotiques de séparation des chromosomes qui ont le plus souvent lieu dans l'ovocyte [1]. Comprendre l'origine de ces défauts est donc un enjeu sociétal. Quels sont donc les mécanismes à l'origine de ce taux élevé d'erreurs chromosomiques dans les ovocytes des mammifères?

\section{Les divisions ovocytaires}

Chez les mammifères, les ovocytes entrent en méiose durant la vie fœtale, avant la naissance, puis se bloquent rapidement en prophase de première division méiotique (prophase I), où ils restent arrêtés pendant de nombreuses années, jusqu'à la reprise de la méiose à partir de la puberté. La méiose s'achève par deux divisions méiotiques (méiose I et II) très asymétriques quant à la taille des cellules filles engendrées, conduisant à la formation d'un gros ovocyte et de deux petits globules polaires qui vont dégénérer (Figure 1). La moitié des chromosomes homologues est expulsée dans les globules polaires, tandis que l'ovocyte conserve l'autre moitié du contenu génomique ainsi que les réserves maternelles indispensables au développement du futur embryon. À la reprise de la méiose, l'enveloppe nucléaire se rompt, et le premier fuseau de division se forme dans la région centrale de l'ovocyte, à l'emplacement du noyau juste avant l'entrée en méiose (Figure I). Puis le fuseau migre progressivement du centre de l'ovocyte vers la zone corticale la plus proche, conduisant à une division asymétrique en taille [1]. L'anaphase I et l'expulsion du premier globule polaire ont lieu environ 8 heures après la rupture de l'enveloppe nucléaire. Puis le fuseau de méiose II se forme parallèlement au cortex, et l'ovocyte s'arrête en
Centre interdisciplinaire de recherche en biologie (CIRB), UMR7241/U1050, Collège de France, 11 place Marcelin Berthelot, 75005 Paris, France.

ismabennabi@gmail.com

marie-emilie.terret@college-de-france.fr

métaphase II (Figure I). L'ovocyte n'est libéré de ce deuxième blocage que par la fécondation, conduisant à l'anaphase II, suivie de l'expulsion du deuxième globule polaire. Débute alors le développement embryonnaire précoce (Figure 1).

\section{Le positionnement du fuseau} de division méiotique

La position du fuseau de division dans la cellule détermine l'endroit où la cellule mère se divise, et constitue donc un élément essentiel de contrôle de la géométrie ainsi que du devenir des cellules filles. Lors de la mitose, les microtubules astraux qui relient les pôles du fuseau de division au cortex cellulaire sont nucléés par les centrosomes et permettent le positionnement du fuseau au sein de la cellule. La transmission efficace des forces de positionnement passe généralement par un ancrage des microtubules astraux à un cortex «dur», conséquence de l'augmentation de la tension corticale à l'entrée en mitose [5].

Les ovocytes des mammifères sont dépourvus de centrosomes canoniques. Chez la souris, ils possèdent, à la place, des centres organisateurs de microtubules acentriolaires (acentriolar micro- 


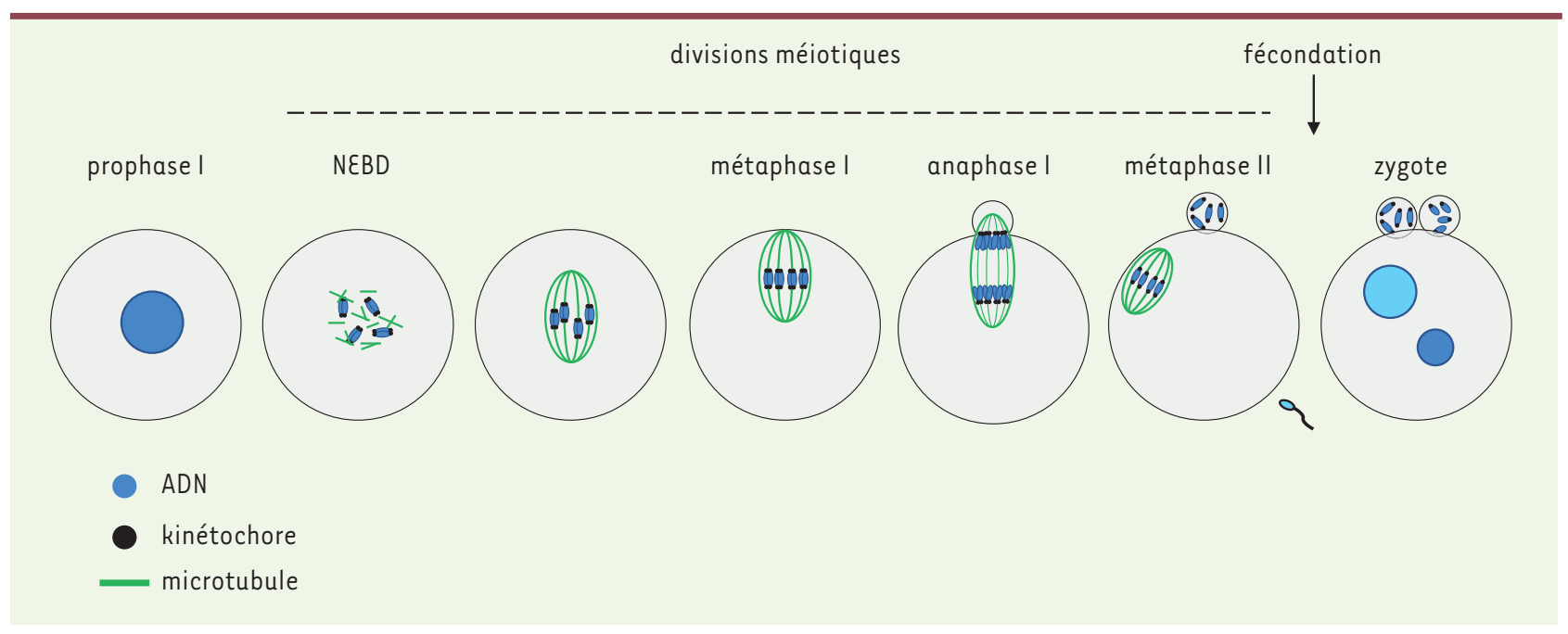

Figure 1. Schéma des divisions méiotiques et du zygote formé par la fécondation de l'ovocyte de souris. Au cours de leur croissance dans l'ovaire, les ovocytes sont arrêtés en prophase I de la méiose. À partir de la puberté, sous l'effet d'une hormone induisant la maturation, les ovocytes reprennent la méiose et enchaînent deux divisions méiotiques asymétriques successives, sans réplication intermédiaire de l'ADN. À l'entrée en première division de méiose (méiose I), l'enveloppe nucléaire se rompt (nuclear envelope breakdown, NEBD), les chromosomes se condensent, et le fuseau de microtubules se forme dans la région centrale de l'ovocyte. Les chromosomes homologues s'alignent progressivement sur la plaque équatoriale du fuseau de division en métaphase I, pendant que le fuseau migre, selon son grand axe, vers la zone du cortex cellulaire la plus proche. Cela permet une division asymétrique en taille après l'anaphase I, qui répartit les chromosomes homologues entre le gros ovocyte et le premier petit globule polaire. L'ovocyte entre directement en seconde division de méiose (méiose II) et le fuseau de microtubules se reforme sous et parallèlement au cortex cellulaire. L'ovocyte reste bloqué en métaphase II, avec les chromosomes alignés sur la plaque équatoriale du fuseau de division. La fécondation induit la sortie de méiose, par une seconde division asymétrique en taille produisant un gros embryon au stade 1-cellule (zygote), et un second petit globule polaire. Les globules polaires vont dégénérer. L’ADN est coloré en bleu, les microtubules en vert, et les kinétochores en noir.

tubule organizing centers, aMTOC) [3, 4], alors que dans l'espèce humaine, aucune structure semblable n'a pu être mise en évidence. Lors de la méiose, le positionnement du fuseau ne dépend pas des microtubules astraux, qui sont absents des pôles des fuseaux méiotiques, mais uniquement de l'actine organisée en deux réseaux distincts. Le premier est un réseau dynamique d'actine cytoplasmique comprenant une cage d'actine entourant le fuseau de microtubules [6-8] (Figure 2). Les nucléateurs d'actine, formine 2 et spirel/2, coopèrent pour assembler les filaments d'actine droits qui composent ce réseau $[9,10]$. Au niveau de la cage d'actine, le moteur moléculaire myosine II exerce des forces de traction sur le cortex ovocytaire, permettant le mouvement du fuseau.

En prophase I, la myosine II est également plus abondante dans le cortex de l'ovocyte, contribuant à l'établissement d'une tension corticale élevée (Figure 2). Un second réseau d'actine est détectable trois heures après la rupture de l'enveloppe nucléaire: un épaississement cortical d'actine branchée nucléé par le complexe Arp2/3 (actin-related proteins 2 et 3) (Figure 2). Cet épaississement cortical d'actine exclut la myosine II corticale, diminuant ainsi la tension corticale $[11,12]$. Contrairement à celui des cellules mitotiques, le cortex de l'ovocyte devient donc «mou» à l'entrée en méiose I. Ce changement de propriétés mécaniques du cortex amplifie un déséquilibre initial des forces exercées par la myosine II au niveau de la cage d'actine, les forces étant probablement plus fortes au pôle du fuseau le plus proche du cortex en raison d'une légère asymétrie initiale dans la position du fuseau. Le déplacement du fuseau est amplifié vers le cortex le plus proche par une déformation progressive du cortex, rendue possible par la baisse de la tension corticale. Ce ramollissement du cortex est indispensable à la migration du fuseau, produisant par conséquent une division asymétrique en taille (Figure 2) [11-13].

\section{Les propriétés mécaniques du cortex de l'ovocyte}

Bien que la baisse de tension corticale soit indispensable à la migration du fuseau dans les ovocytes, celle-ci est également empêchée par une tension corticale trop basse ou trop élevée [2, 12]. Ainsi, la géométrie de la division dépend d'une fenêtre étroite de tension corticale, contrôlée par la localisation au cortex de la myosine $I I$, elle-même contrôlée par la nucléation d'actine à cet emplacement. De plus, il a été récemment montré que les propriétés mécaniques du cortex cellulaire peuvent prédire le potentiel développemental des ovocytes après fécondation chez la souris et chez l'homme [14]. En effet, 


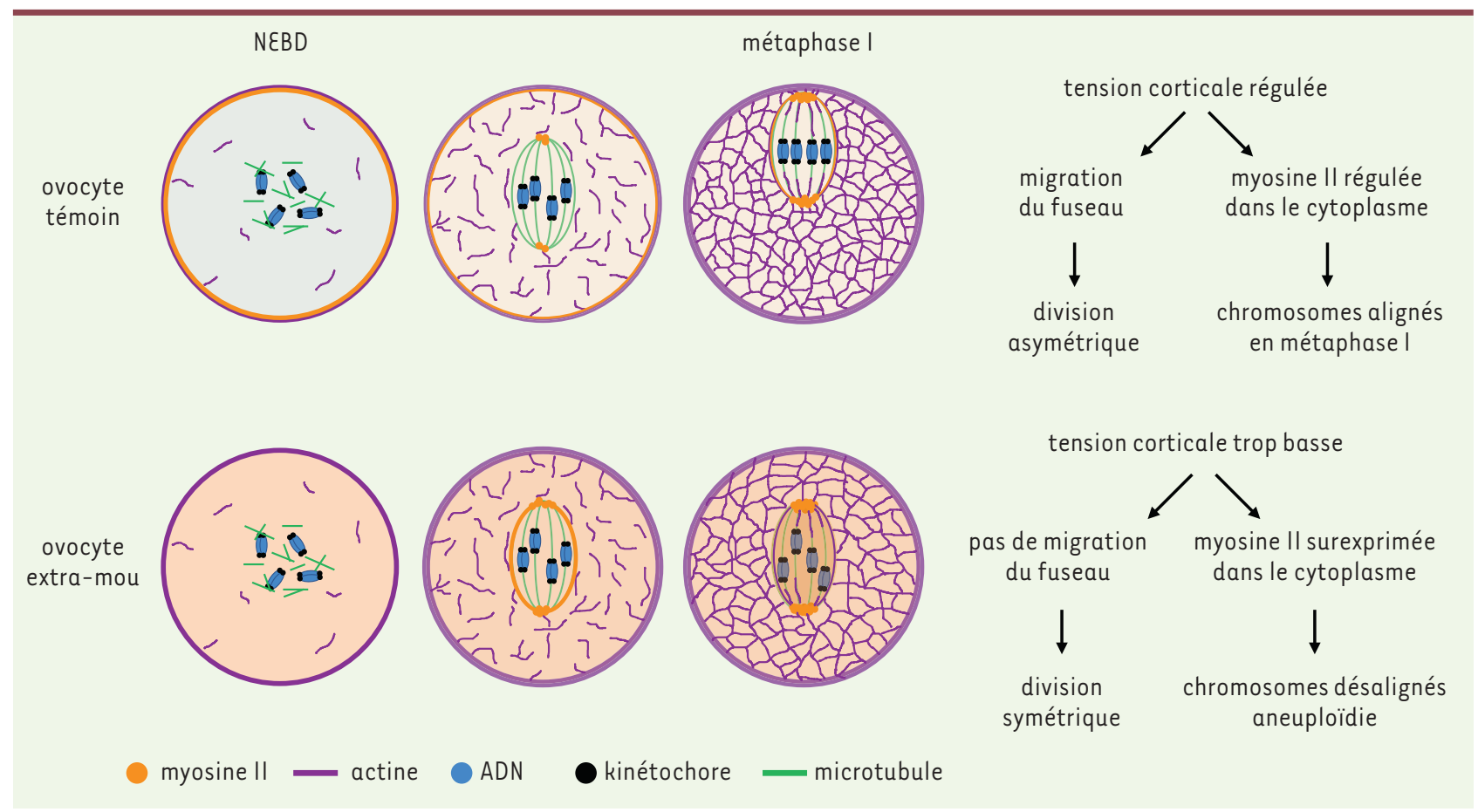

Figure 2. Phénotype des ovocytes trop mous. Dans un ovocyte témoin en prophase I, le cortex cellulaire est enrichi en myosine II, contribuant à une tension corticale élevée. Après la rupture de l'enveloppe nucléaire (nuclear envelope breakdown, NEBD), deux réseaux d'actine se mettent progressivement en place, tous deux essentiels à la migration du fuseau du centre de l'ovocyte vers le cortex cellulaire. Le premier est un réseau cytoplasmique comprenant une cage d'actine entourant le fuseau de microtubules, sur laquelle la myosine II exerce des forces de traction. Le second est un épaississement cortical d'actine, qui exclut la myosine II du cortex, diminuant ainsi la tension corticale. Le cortex de l'ovocyte en méiose I devient donc mou. II est possible d'obtenir des ovocytes trop mous, un défaut relativement fréquent dans une population naturelle d'ovocytes murins et humains. Les ovocytes trop mous chassent trop précocement la myosine II du cortex cellulaire, dès la prophase I. La myosine II s'accumule alors dans le cytoplasme et le fuseau de l'ovocyte, ce qui gêne la capture des chromosomes par le fuseau, entraînant de l'aneuploïdie. L'ADN est coloré en bleu, les microtubules en vert, les kinétochores en noir, la myosine II en orange, et l'actine en violet.

les ovocytes et embryons présentant un cortex cellulaire trop dur ou trop mou ne se développent pas après le stade blastocyste. Quelles sont donc les causes de l'arrêt du développement précoce dû à des défauts de tension corticale dans l'ovocyte?

\section{Les ovocytes trop mous sont aneuploïdes}

Les ovocytes dont la tension corticale est trop basse représentent le cas le plus fréquent dans une population naturelle d'ovocytes murins et humains. Afin d'en augmenter encore le nombre, nous avons développé des outils capables de diminuer la tension corticale. Nous avons fait surexprimer par l'ovocyte une construction, le cVCA, dans laquelle le domaine verprolin central acidic
(VCA) de la protéine WAVE2 (WiskottAldrich syndrome protein family verprolin-homologous), facteur favorisant la nucléation d'actine par le complexe Arp2/3, est fusionné à la protéine corticale ezrine [12, 15]. L'expression de cette construction par les ovocytes de souris active localement le complexe Arp2/3 au cortex cellulaire, et en chasse précocement la myosine II en prophase I (Figure 2). L'exclusion de la myosine II du cortex cellulaire par l'épaississement de l'actine corticale entraîne une diminution de la tension corticale, comme cela a été montré par la technique d'aspiration à l'aide de micropipettes [12, $13,15]$. Les ovocytes exprimant le cVCA sont donc très mous. Ces ovocytes présentent un taux élevé d'erreurs d'alignement des chromosomes, ce qui entraîne de l'aneuploïdie (gain ou perte d'un chromosome entier) après l'anaphase I (Figure 2) [15].

Par ailleurs, une analyse approfondie du comportement des chromosomes au cours de la méiose I a montré qu'ils «explorent» plus d'espace dans ces ovocytes «extra-mous», ce qui pourrait indiquer un défaut de capture des chromosomes homologues par les microtubules du fuseau. Une mesure indirecte de la tension entre les chromosomes homologues attachés par leurs kinétochores au fuseau de microtubules indique que les chromosomes sont moins sous tension. Ce résultat est compatible avec le fait que les chromosomes sont capturés moins efficacement par le fuseau dans les ovocytes extra-mous (Figure 2) [15]. 
Pour mieux comprendre l'origine de ces défauts chromosomiques, nous avons observé la morphogenèse du fuseau de microtubules dans les ovocytes extramous. En effet, dans les cellules mitotiques, une tension corticale aberrante altère la formation du fuseau, entraînant des erreurs de ségrégation des chromosomes. Étonnamment, la morphogenèse du fuseau dans les ovocytes extra-mous est comparable à celle d'ovocytes témoins [15]. Comment alors expliquer ce taux élevé d'erreurs chromosomiques dans les ovocytes présentant une tension corticale très basse?

\section{L'accumulation de myosine II} dans l'ovocyte génère des erreurs chromosomiques

La myosine II étant chassée précocement du cortex cellulaire dans les ovocytes extra-mous, son accumulation ectopique dans le cytoplasme pourrait gêner la capture des chromosomes par les microtubules. Pour tenter de comprendre les mécanismes empêchant la capture et l'alignement des chromosomes, nous avons suivi la myosine II au cours de la méiose I. Nous avons montré que les niveaux de myosine II endogène et active sont plus élevés dans le cytoplasme et sur le fuseau de microtubules dans les ovocytes extra-mous que dans des ovocytes témoins (Figure 2) [15]. De plus, les ovocytes extra-mous traités avec un inhibiteur de l'activation de la myosine II ne présentent plus ces défauts d'alignement des chromosomes [15]. Ainsi, l'accumulation anormale de la myosine II dans le cytoplasme et sur le fuseau des ovocytes extra-mous pourrait indirectement perturber la capture des chromosomes.

\section{Perspectives}

L'alignement des chromosomes est donc sévèrement altéré dans les ovocytes présentant une tension corticale trop basse, du fait d'une anomalie de répartition de la myosine II : celle-ci se dissocie précocement du cortex cellulaire, induisant une forte diminution de la tension corticale, et sa concentration globale augmente dans le cytoplasme. Sa fixation aux chromosomes pourrait créer un encombrement stérique local, empêchant la capture des chromosomes et conduisant à des défauts d'alignement et de ségrégation des chromosomes. Ces résultats décrivent un mode de production d'aneuploïdie qui pourrait être très courant dans les gamètes femelles. En effet, $36 \%$ des ovocytes d'une population naturelle d'ovocytes murins et humains sont mesurés comme «trop mous » [14]. Ainsi, certains de ces ovocytes «naturellement mous » pourraient également présenter des défauts chromosomiques entravant leur développement futur après la fécondation, et contribuant au taux d'aneuploïdie élevé observé dans les gamètes femelles. Les mesures des propriétés mécaniques du cortex cellulaire pourraient donc servir à évaluer, par une technique non invasive, le potentiel développemental des ovocytes dans le cadre de la procréation médicalement assistée. $\diamond$

Cortical tension of the oocyte and euploidy: the right balance

\section{REMERCIEMENTS}

Nous remercions les co-auteurs de la publication originale. Ces travaux ont bénéficié du soutien logistique et financier de la Fondation pour la recherche médicale (MHV-DEQ20150331758 puis EQU201903007796), de l'ANR (ANR-14-CE11 MHV, ANR-16-CE13 MET) et du Labex Memolife de PSL. Ce travail a reçu le soutien de la fondation
Bettencourt Schueller, programme Investissements d'avenir (ANR-10-LABX-54 MEMO LIFE, ANR-11IDEX-0001-02 PSL* Research university).

\section{LIENS D'INTÉRÊT}

Les auteures déclarent n'avoir aucun lien d'intérêt concernant les données publiées dans cet article.

\section{RÉFÉRENCES}

1. Nagaoka SI, Hassold TJ, Hunt PA. Human aneuploidy: mechanisms and new insights into an age-old problem. Nat Rev Genet 2012 ; 13 : 493-504.

2. Verlhac MH, Lefebvre C, Guillaud P, et al. Asymmetric division in mouse oocytes: with or without Mos. Curr Biol 2000 ; 10 : 1303-6.

3. Szollosi D, Calarco P, Donahue RP. Absence of centrioles in the first and second meiotic spindles of mouse oocytes. J Cell Sci 1972 ; 11 : 521-41.

4. Manandhar $G$. Centrosome reduction during gametogenesis and its significance. Biol Reprod $2005 ; 72: 2-13$.

5. Kunda P, Pelling AE, Liu T, Baum B. Moesin controls cortical rigidity, cell rounding, and spindle morphogenesis during mitosis. Curr Biol 2008; 18 : 91-101.

6. Azoury J, Lee KW, Georget V, et al. Spindle positioning in mouse oocytes relies on a dynamic meshwork of actin filaments. Curr Biol 2008 ; 18 : 1514-9.

7. Schuh M, Ellenberg J. A new model for asymmetric spindle positioning in mouse oocytes. Curr Biol 2008 ; 18 : 1986-92

8. Mogessie B, Schuh M. Actin protects mammalian eggs against chromosome segregation errors. Science 2017 ; 357 : eaall647.

9. Pfender S, Kuznetsov V, Pleiser S, et al. Spire-type actin nucleators cooperate with formin-2 to drive asymmetric oocyte division. Curr Biol $2011 ; 21$ : 955-60.

10. Almonacid M, Ahmed WW, Bussonnier M, et al. Active diffusion positions the nucleus in mouse oocytes. Nat Cell Biol $2015 ; 17: 470-9$.

11. Chaigne A, Campillo C, Gov NS, et al. A soft cortex is essential for asymmetric spindle positioning in mouse oocytes. Nat Cell Biol $2013 ; 15$ : 958-66.

12. Chaigne A, Campillo C, Gov NS, et al. A narrow window of cortical tension guides asymmetric spindle positioning in the mouse oocyte. Nat Commun 2015; 6: 6027.

13. Larson SM, Lee HJ, Hung P, et al. Cortical mechanics and meiosis II completion in mammalian oocytes are mediated by myosin-II and ezrin-radixin-moesin (ERM) proteins. Mol Biol Cell $2010 ; 21$ : 3182-92.

14. Yanez LZ, Han J, Behr BB, et al. Human oocyte developmental potential is predicted by mechanical properties within hours after fertilization. Nat Commun 2016; $7: 10809$.

15. Bennabi I, Crozet F, Nikalayevich $\varepsilon$, et al. Artificially decreasing cortical tension generates aneuploidy in mouse oocytes. Nat Commun $2020 ; 11: 1649$.

\section{Retrouvez toutes les Actualités de la Myologie sur les sites de :}
la Société Française de Myologie
www.sfmyologie.org
sfm
la filière de santé neuromusculaire FILNEMUS
www.filnemus.fr

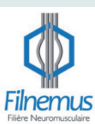

